\title{
Interplay of the ubiquitin proteasome system and the innate immune response is essential for the replication of infectious bronchitis virus
}

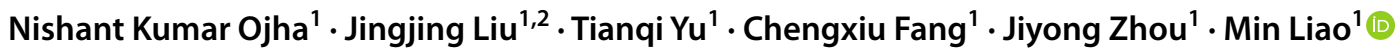

Received: 17 December 2020 / Accepted: 3 March 2021 / Published online: 26 May 2021

(c) The Author(s), under exclusive licence to Springer-Verlag GmbH Austria, part of Springer Nature 2021

\begin{abstract}
Infectious bronchitis virus (IBV) is the only coronavirus known to infect poultry. The replication and pathogenesis of IBV are poorly understood, mainly because of the unavailability of a robust cell culture system. Here, we report that an active ubiquitin proteasome system (UPS) is necessary for efficient replication of IBV in Vero cells. Synthesis of IBV-specific RNA as well as viral protein is hampered in the presence of chemical inhibitors specific for the UPS. Like other coronaviruses, IBV encodes a papain-like protease (PLpro) that exhibits in vitro deubiquitinase activity in addition to proteolytically processing the replicase polyprotein. Our results show that the IBV PLpro enzyme inhibits the synthesis of interferon beta (IFN $\beta$ ) in infected chicken embryonic fibroblast (DF-1) cells and that this activity is enhanced in the presence of melanoma differentiation-associated protein 5 (MDA5) and TANK binding kinase 1 (TBK1). IBV PLpro, when overexpressed in DF-1 cells, deubiquitinates MDA5 and TBK1. Both of these proteins, along with other adapter molecules such as MAVS, IKKe, and IRF3, form a signaling cascade for the synthesis of IFN $\beta$. Ubiquitination of MDA5 and TBK1 is essential for their activation, and their deubiquitination by IBV PLpro renders them unable to participate in antiviral signaling. This study shows for the first time that there is cross-talk between the UPS and the innate immune response during IBV infection and that the deubiquitinase activity of IBV PLpro is involved in its activity as an IFN antagonist. This insight will be useful for designing better antivirals targeting the catalytic activity of the IBV PLpro enzyme.
\end{abstract}

\section{Introduction}

Infectious bronchitis virus (IBV) is a highly contagious pathogen that infects poultry globally, causing heavy economic losses. IBV is transmitted via the aerial route and causes destruction of the tracheal epithelium of chickens, making them susceptible to secondary bacterial infections. Later, the virus also spreads to other organs, including the gastrointestinal tract, kidney, and oviduct, thereby affecting egg quality $[1,2]$. IBV is the prototype member of the genus Gammacoronavirus, family Coronaviridae, order Nidovirales. Its

Handling Editor: Sheela Ramamoorthy.

Min Liao

liaomin4545@zju.edu.cn

1 Key Laboratory of Animal Virology of Ministry of Agriculture, Department of Veterinary Medicine, College of Animal Science, Zhejiang University, 866 Yuhangtang Road, Hangzhou, People's Republic of China

2 Yinhe Biomedical Investment Co., Ltd, Beihai, Guangxi, People's Republic of China genome is a positive-sense, nonsegmented, single-stranded RNA, $\sim 27.6 \mathrm{~kb}$ in size [3]. Approximately two-thirds of the genome at the 5' end consists of open reading frame 1 , which, due to ribosomal frameshifting, is composed of orf1a and orf $1 \mathrm{~b}$. The remaining one-third of the genome at the 3' end consists of structural and accessory genes in the following order: spike (S), accessory genes $3 \mathrm{a}$ and $3 \mathrm{~b}$, envelope (E), membrane (M), accessory genes $5 \mathrm{a}$ and $5 \mathrm{~b}$, and nucleocapsid $(\mathrm{N})$. All of the nonstructural proteins (2 to 16) are encoded by orf1 and together form the replication/transcription complex. ORF1a encodes a 3951-aminoacid-long polyprotein from which individual viral proteins are generated by proteolytic processing by cellular and viral proteases [4].

Nonstructural protein 3 of IBV strain M41 (GenBank no. DQ834384.1), corresponding to amino acids 11741481 in ORF1a, is called the papain-like protease (PLpro) domain, and it has considerable sequence similarity to the PLpro proteins of other coronaviruses [5]. IBV PLpro has been shown to have proteolytic activity in vitro, and it plays a role in the processing of the ORF1 polyprotein [6]. In addition to its proteolytic function in the viral replicase 
complex, PLpro also possesses deubiquitinase activity and can degrade Lys48- and Lys63-linked polyubiquitin chains to monoubiquitin, but not linear polyubiquitin [7, 8]. Acute infection with IBV elicits a type 1 innate immune response, and there is a significant upregulation of expression of IFN $\beta$, IRF3, melanoma differentiation-associated protein5 (MDA5), and Toll-like receptor 3 (TLR3), which suggests the involvement of the TLR3 pathway upon IBV infection [9]. MDA5 is the major pattern recognition receptor for the IBV double-strand RNA intermediate, which together with mitochondrial antiviral signaling (MAVS), acts as a positive regulator of the type 1 interferon response against IBV infection [10]. The ubiquitin proteasome system (UPS) is a major pathway of protein degradation in eukaryotic cells. It consists of ubiquitin conjugation and substrate degradation machinery and plays important role during viral infection [11]. The posttranslational modification of viral proteins by the host cell UPS is a major way of regulating and inhibiting their functions. However, viruses can employ several strategies to subvert this effect. The UPS modulates the cellular innate immune response against viral infection [12]. TRIM65 is involved in K63-linked ubiquitination at lysine 743 of MDA5, which is essential for MDA5 oligomerization and activation [13]. TBK1 has a ubiquitin-like domain (ULD) adjacent to its kinase domain, which is necessary for the IRF3 phosphorylation and nuclear localization that are required for IFN $\beta$ synthesis [14]. Deubiquitination of MDA5, TBK1, and other proteins involved in the innate immune response by viral proteins is one of the mechanisms by which of the virus survives in host cells [15].

The aim of present study was to elucidate the underlying mechanism by which IBV interferes with type 1 IFN induction. We show that IBV replication in Vero cells is inhibited in the presence of specific chemical inhibitors of the ubiquitin proteasome system and that the IBV PLpro domain plays a specific role in deubiquitinating host proteins related to innate immunity. IBV PLpro deubiquitinates MDA5 and TBK1, whose activity is necessary for eliciting a type 1 interferon response, and thereby acts as an IFN antagonist. Cross-talk between UPS and the type 1 innate immune response is essential for the replication of IBV in host cells.

\section{Materials and methods}

\section{Cell culture, virus, and plasmids}

The chicken embryonic fibroblast cell line DF-1 (ATCC; CRL-12203) and the African green monkey kidney cell line Vero (ATCC; CCL-81) were maintained in Dulbecco's modified Eagle's medium (Gibco, Carlsbad, CA) supplemented with $10 \%$ fetal bovine serum (FBS), penicillin (100 units/
$\mathrm{mL})$, and streptomycin $(100 \mu \mathrm{g} / \mathrm{mL})$ at $37^{\circ} \mathrm{C}$ in a humidified chamber with a $5 \% \mathrm{CO}_{2}$ environment. For Vero cells, we used FBS (Biological Industries; cat. no. 1616756), and for DF-1 cells, serum (MRC; cat. no. CCS30010.02) was used. The Vero-adapted IBV Beaudette strain [6] was a kind gift from Dr. Dingxiang Liu (South China Agricultural University, Guangzhou, P. R. of China). The IBV Massachusetts strain (M41) (GenBank: no. MK937830.1) was purchased from China Institute of Veterinary Drug Control and maintained in our laboratory. The Beaudette and M41 strains of IBV have 95\% amino acid sequence identity in their spike (S1) protein and belong to the same Massachusetts serotype [16]. Virus stocks (Beaudette $\log _{10} \mathrm{FFU} / \mathrm{mL}=7.84$ and M41 $\log _{10} \mathrm{FFU} / \mathrm{mL}=4.34$ ) were stored at $-80{ }^{\circ} \mathrm{C}$ until they were used. Mammalian expression vectors pCMV(DYKDDDDK)-N (cat. no. 635688) and pCMV-Myc-N (cat. no. 635689) were purchased from Clontech Laboratories (Mountain View, CA), and restriction sites for the enzymes EcoR I and Kpn I in their multiple cloning sites were used to clone chicken TBK1 (NCBI reference sequence NM_001199558.1) and the IBV M41 PLpro domain, respectively (for primer sequences, see Table 1). The plasmids pCMV-HA-Ub, pGL3-chIFN- $\beta$, and pCMV-N-Flag-MDA5 were already available in our laboratory $[17,18]$.

\section{Reagents and antibodies}

Inhibitor compounds, including MG132 (Beyotime Biotechnology; cat. no. S1748), lactacystin (APExBIO; cat no. A2583), and epoxomicin (AbMole BioScience; cat. no. M2193) were dissolved in DMSO at a concentration of 100 $\mu \mathrm{M}$ and stored at $-20{ }^{\circ} \mathrm{C}$ until further use. Radioimmunoprecipitation assay (RIPA) buffer (Beyotime Biotechnology; cat. no. P0013B), nitrocellulose blotting membranes (GE Healthcare; cat. no. 10600002), jetPRIME transfection reagent (Polyplus Transfection S.A; cat. no. 712-60 and 114-15), and chemiluminescent Western blotting detection reagent Super Western ECL were obtained from BioBEST company, and poly(I:C) was purchased from InvivoGen.

Antibodies against c-Myc tag (Huabio Inc.; cat. no. 1208-1), Flag (DYKDDDDK) tag (Sigma-Aldrich; cat. no. F1804), HA-tag (Sigma-Aldrich, cat. no. 9658), anti-actin (Abcam; cat. no. ab179467), anti-ubiquitin (Cell Signaling Technology; cat. no. 3936S), anti-mouse IgG HRP (Bioker Biotech; cat. no. 074-1806), anti-rabbit IgG HRP (Bioker Biotech; cat. no. 074-1506), and anti-mouse IgG FITC (KPL; cat. no. 5230-0427) were used. Monoclonal antibodies against the IBV membrane protein and nucleocapsid protein and polyclonal rabbit sera against chicken IRF3 were prepared previously in our laboratory. 
Table 1 Primers

\begin{tabular}{lll}
\hline Gene name & Nucleotide sequence (5'-3') \\
\hline IBV nucleocapsid & F & AGGGCTCTCGCATTACCAAG \\
& R & GAGCATTGCTGTAACACGCC \\
Monkey GAPDH & F & CATGAGAAGTATGACAACAGCCT \\
& R & AGTCCTTCCACGATACCAAAGT \\
IBV PLpro & F & CATCGAATTCACGCCACCATGGGAACTTGTAAGCAGAAGACAAT \\
& R & CATCGGTACCTCAATGTTCAACAACAAGTAGGGGATTTCACTC \\
Chicken IFN $\beta$ & F & GCTCTCACCACCACCTTCTC \\
& R & GCTTGCTTCTTGTCCTTGCT \\
Chicken TBK1 & F & CATCGAATTCACGCCACCATGGGAATGCAGAGCACCTCGAATTACCTC \\
& R & CATCGGTACCTTAGATACAGTCCACATTCCTCAGGCCACCATCTGC \\
Chicken GAPDH & F & CATCACAGCCACACAGAAG \\
& R & GGTCAGGTCAACAACAGAGA \\
\hline
\end{tabular}

\section{Reverse transcription quantitative PCR}

Vero and DF-1 cells were cultured in a 24-well plate to $70 \%$ confluence and then infected with the IBV strain Beaudette (MOI of 1) and M41 (MOI of 10), respectively. At the indicated time points, cells were lysed with SuPerfecTRI lysis reagent (Pufei; cat. no. 3101) for RNA extraction. Quantification of RNA was done using a NanoDrop spectrophotometer (Thermo Scientific). Synthesis of cDNA was done using HiScript II Q RT Supermix reverse transcriptase (Vazyme Biotech; cat. no. R222-01) according to the manufacturer's instructions. Expression levels of RNA transcripts for the IBV nucleocapsid and IFN $-\beta$ genes were measured using AceQ qPCR reagent (Vazyme Biotech; cat no. 111-02) in a LightCycler 96 System (Roche). Genes of monkey GAPDH and chicken GAPDH were used as input controls (for primer sequences, see Table 1). The fold change in gene expression was calculated by the $2^{-\Delta \Delta \mathrm{Ct}}$ method as described previously [19].

\section{Protein extraction and immunoblot}

Cultured cells were washed three times with ice-cold Dulbecco's phosphate-buffered saline (PBS) and then lysed in ice-cold RIPA buffer supplemented with phenylmethylsulfonyl fluoride (PMSF; protease inhibitor). The crude protein was then extracted by centrifuging the lysate at $\sim 13,000 \times$ $g$ for 10 minutes at $4{ }^{\circ} \mathrm{C}$. The supernatant was collected in a separate microfuge tube. Protein samples were subjected to SDS-polyacrylamide gel electrophoresis and transferred to a nitrocellulose membrane. The membrane was blocked in 5\% skimmed milk powder dissolved in PBS for $1 \mathrm{~h}$ at $37^{\circ} \mathrm{C}$ in an orbital shaker, after which the membranes were washed three times with PBS- $0.1 \%$ Tween 20 (PBS-T), followed by incubation with primary antibody (1:1000 dilution) for $2 \mathrm{~h}$ at $37{ }^{\circ} \mathrm{C}$. The membranes were again washed three times with PBS-T at $37{ }^{\circ} \mathrm{C}$ and incubated with anti-mouse $\operatorname{IgG}$ HRP-conjugated secondary antibody (1:5000 dilution) for $1 \mathrm{~h}$ at $37^{\circ} \mathrm{C}$. After the second incubation, membranes were again washed three times with PBS-T. Finally, immunoreactive protein bands on the membrane were visualized using enhanced chemiluminescence reagent, and images were captured using an AI680 Imager (GE Healthcare).

\section{Immunoprecipitation assay}

All immunoprecipitation experiments were conducted in DF-1 cells cultured in 6-well plates. When cells attained $70 \%$ confluence, they were infected with IBV strain M41 at an MOI of 10, and after $24 \mathrm{~h}$ of infection, the cells were transfected with either pCMV-N-Flag-MDA5 or pCMV-NFlag-TBK1. For co-immunoprecipitation assays, the cells were cotransfected with pCMV-N-myc-IBV PLpro and pCMV-N-Flag-MDA5 or pCMV-N-Flag-TBK1. After $48 \mathrm{~h}$ of transfection, cells were washed three times with ice-cold PBS and then harvested in lysis buffer ( $50 \mathrm{mM}$ Tris- $\mathrm{Cl}, \mathrm{pH}$ 8.0, $150 \mathrm{mM} \mathrm{NaCl}, 1 \%$ Tween-20, $0.1 \%$ SDS) supplemented with protease inhibitor. Protein samples were incubated for $2 \mathrm{~h}$ at $4{ }^{\circ} \mathrm{C}$ with an anti-Flag antibody. After that, the lysateantibody complex was captured on Protein A/G PLUS-Agarose beads (Santa Cruz Biotechnology Inc.; cat no. sc-2003) by overnight incubation at $4{ }^{\circ} \mathrm{C}$ in an orbital shaker. Beads were washed three times with PBS-T and then suspended in SDS-PAGE sample loading buffer. Precipitated proteins were eluted by boiling the beads for 5 minutes. Eluted protein samples were detected by Western blot.

\section{Indirect immunofluorescence assay}

Cells were cultured in 96-well plates and infected with IBV. At the indicated time points, the cells in each well were fixed and permeabilized by incubating them with $100 \mu \mathrm{L}$ of 
methanol:acetone at a ratio of $1: 1$ for 30 minutes at $-20{ }^{\circ} \mathrm{C}$. They were then blocked with $100 \mu \mathrm{L}$ of $5 \%$ skimmed milk powder for $1 \mathrm{~h}$ at room temperature (RT) and washed three times with PBS-T. They were then incubated with an mAb against the IBV membrane protein (1:1000) for $1 \mathrm{~h}$ at RT in an orbital shaker and washed three times with PBS-T. Subsequently, the cells were incubated with FITC-conjugated anti-mouse IgG secondary antibody (1:500) for $1 \mathrm{~h}$ at RT in the dark. After the incubation, the cells were washed three times with PBS-T and then visualized under a fluorescent microscope. Fluorescent foci were counted, and the number of focus-forming units (FFU) in each well was calculated as described previously [20].

\section{Cell viability and dual luciferase reporter assay}

DF-1 cells in each well of a 48-well plate were transfected with $200 \mathrm{ng}$ of pGL3-chIFN- $\beta$, together with $200 \mathrm{ng}$ of the plasmids indicated below and $10 \mathrm{ng}$ of the internal control plasmid pRL-TK or $400 \mathrm{ng}$ of pGL3-chIFN- $\beta$ with $10 \mathrm{ng}$ of pRL-TK only. A lysate of the cells transfected with the empty vector was used as a control for calculation of the IFN $\beta$ promoter activation level. Luciferase activity in the samples was measured using a Dual Luciferase Assay Kit (Beyotime Biotechnology; cat. no. RG027) according to the manufacturer's instructions in a Spectra Max M5 microplate reader (Molecular Devices, CA). The relative firefly luciferase activity is shown after normalization with the Renilla luciferase level.

A cell viability assay was conducted in a 96-well plate using an alamarBlue assay (Solarbio Life Science; cat. no. A7631) according to the manufacturer's instructions. Resazurin (alamarBlue) is a cell-permeable non-fluorescent indigo blue dye that, upon entering a cell, gets reduced to fluorescent resorufin. Viable cells continuously convert resazurin to resorufin, and the resulting fluorescence is measured at an excitation wavelength of $560 \mathrm{~nm}$ and an emission wavelength of $590 \mathrm{~nm}$. The assay was performed to measure the toxicity of UPS inhibitors on Vero cells and of the IBV PLpro clone construct on DF-1 cells.

\section{Statistical analysis}

All experimental data sets were at least in triplicate. Statistical analysis was conducted using a two-tailed, unpaired Student's $t$-test using SPSS statistics software. Differences were considered significant at a value of $*, p<0.05$; **, $p$ $<0.01$; or $* * *, p<0.005$. Fold change in signal intensity of Western blot bands were determined by measuring their densitometric values with ImageJ v1.50b software (NIH, USA), and figures were prepared using the MS Office suite.
Fig. 1 Effects of proteasome inhibitors on IBV Beaudette strain infection in Vero cells. Vero cells were treated with proteasome inhibitors at the indicated concentrations. (a) The cell viability after treatment was assessed using an alamarBlue assay. (b) IBV genomic copies in inhibitor-treated Vero cells. After infection with IBV at an MOI of 1 , cells were incubated with DMEM containing proteasome inhibitors. The supernatant was collected at $72 \mathrm{hpi}$, and the number of IBV genomic copies was determined by RT-qPCR. The graph represents the averages of three independent experiments. (c) Indirect immunofluorescence assay (IFA) was performed after $72 \mathrm{~h}$ of infection using an antibody against the IBV membrane protein. The test and mock-treated cells were stained in triplicate, and representative images are shown. (d) Fluorescent foci in an indirect IFA experiment were counted, and the virus titer was determined. (e) An immunoblot of the whole-cell lysate was performed using an antibody against the IBV nucleocapsid protein. Actin from the same protein samples served as a loading control. (f) Overexpression of ubiquitin by transfection with pCMV-HA-Ubiquitin partially restored IBV replication, which was inhibited in the presence of MG132. The error bars indicate standard error. Significant differences between test samples and controls are indicated by asterisks $(* *, p \leq 0.01)$

\section{Results}

\section{IBV replication is inhibited by specific chemical inhibitors of the ubiquitin proteasome system}

The ubiquitin proteasome system plays a critical role at different stages of the infection cycle of several coronaviruses $[21,22]$. To study the role of the UPS in IBV replication, we used three different UPS inhibitors, namely, MG132, lactacystin, and epoxomicin. First, we tested the cytotoxicity of the three inhibitors on cell growth by alamarBlue assay [23], and we found that $>90 \%$ of Vero cells survived in the presence of 1-3 $\mu \mathrm{M}$ MG132, lactacystin, and epoxomicin (Fig. 1a) after $72 \mathrm{~h}$ of supplementation. Therefore, a concentration of 1-3 $\mu \mathrm{M}$ of these three inhibitors was selected for further experiments. We infected Vero cells with the IBV Beaudette strain at a MOI of 1 for $72 \mathrm{~h}$ and measured virus replication by quantitative PCR (qPCR), Western blot, and indirect IFA. We measured the level of IBV nucleocapsid protein in the cell culture supernatant by qPCR, and in whole cell lysates by Western blot, and in situ expression of the IBV membrane protein was detected by indirect IFA. We found that all three inhibitors were effective in inhibiting IBV replication. The qPCR results showed that, after $72 \mathrm{~h}$, there was a significant reduction (nearly half at $3 \mu \mathrm{M}$ ) in the virus copy number (Fig. 1b). Similar results were obtained by IFA for the membrane protein and immunoblot for the nucleocapsid protein (Fig. 1c and e). Virus titration showed that there was a dose-dependent reduction in the number of fluorescent foci when cells were treated with inhibitors (Fig. 1d). Previously, it was reported that MG132 reduces the pool of free ubiquitin inside the cells [24]. Hence, to confirm that the observed inhibition of IBV replication by MG132 was due to the depletion of free ubiquitin, we transfected 


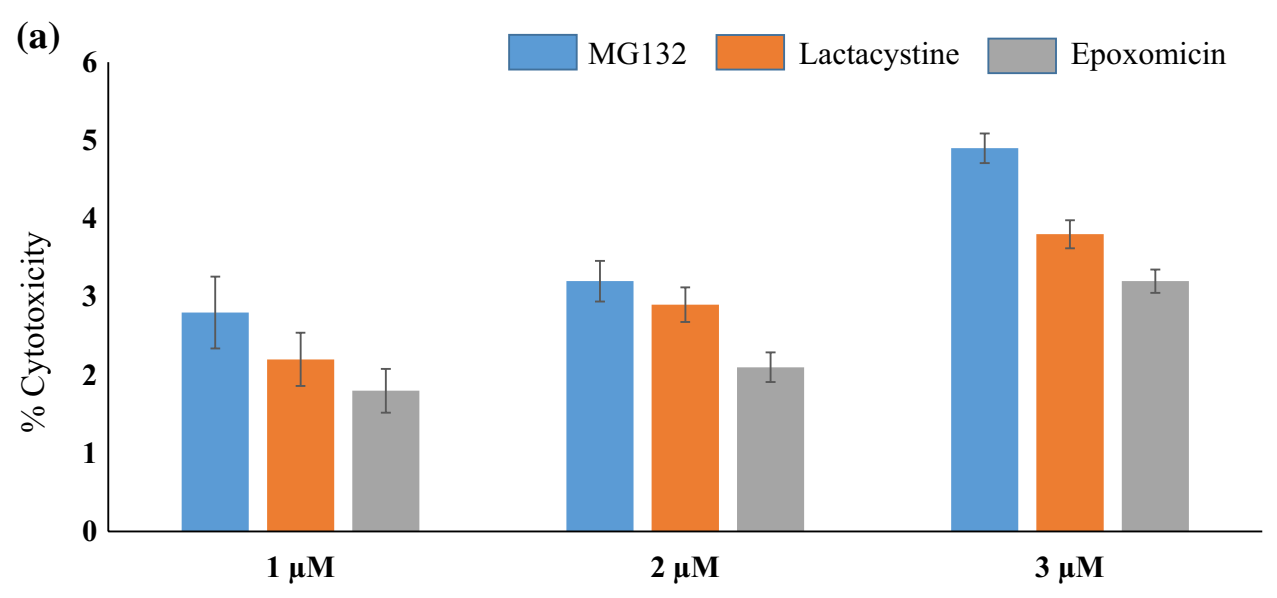

(b)

Chemical concentration

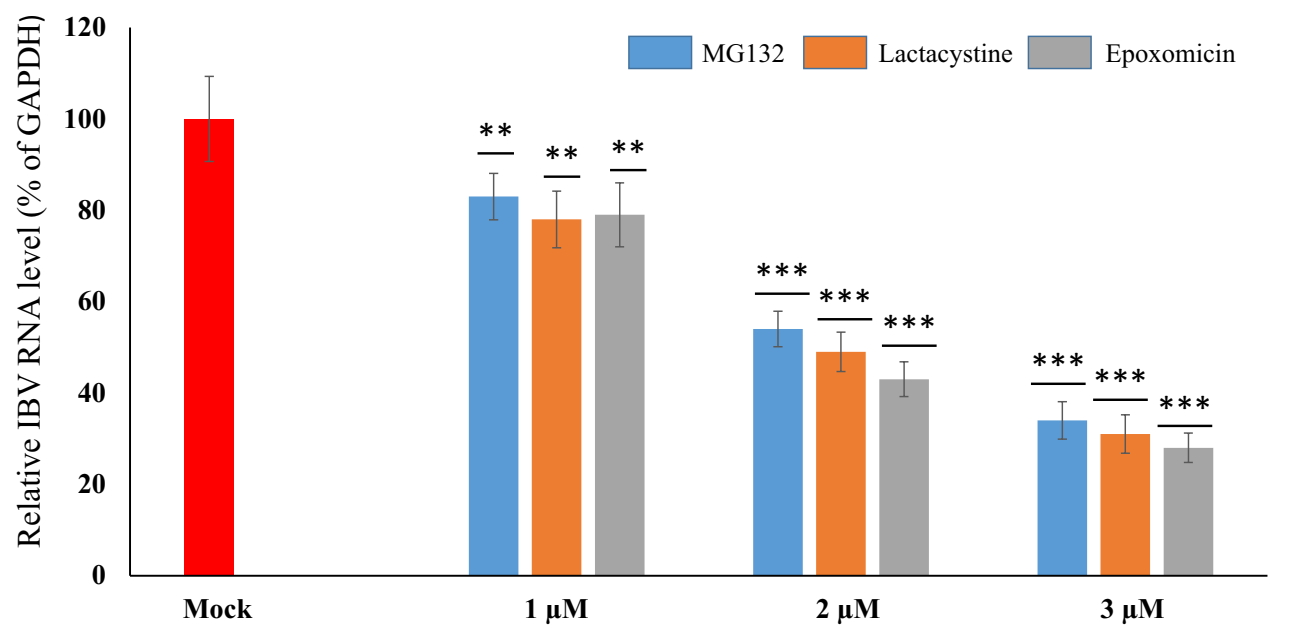

Chemical concentration
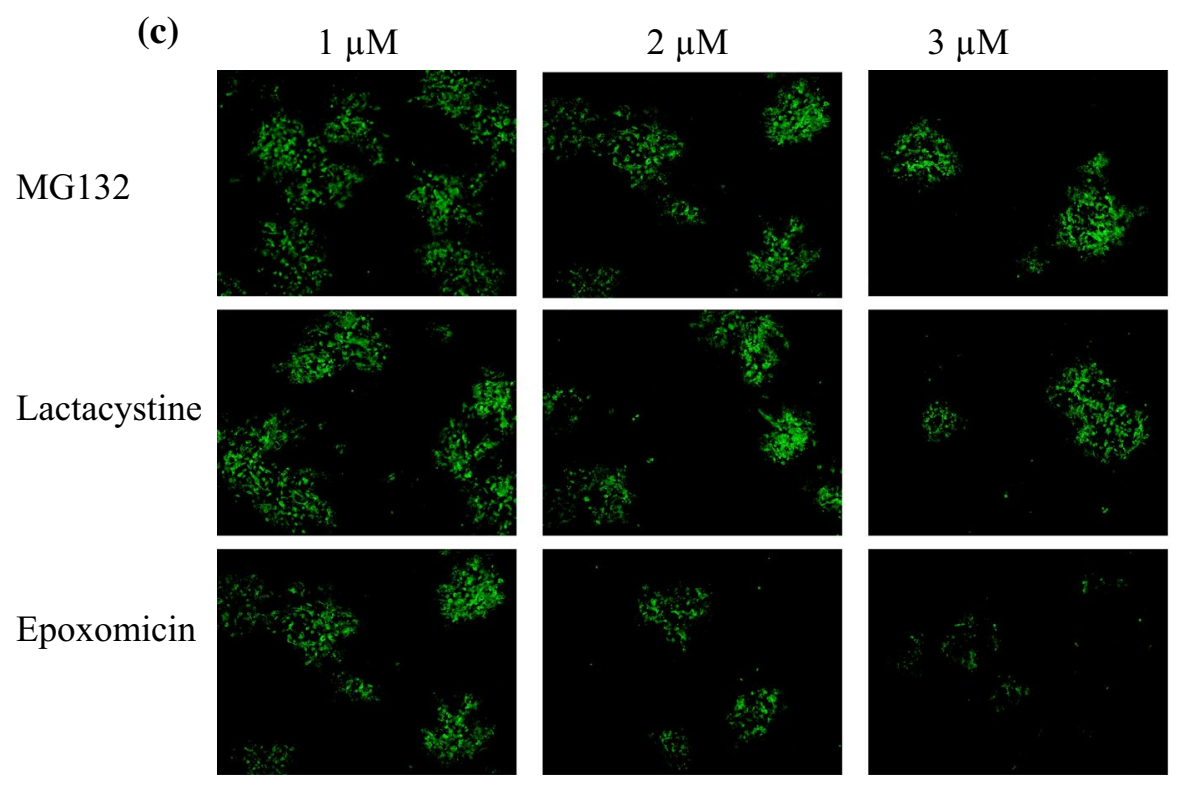

Mock
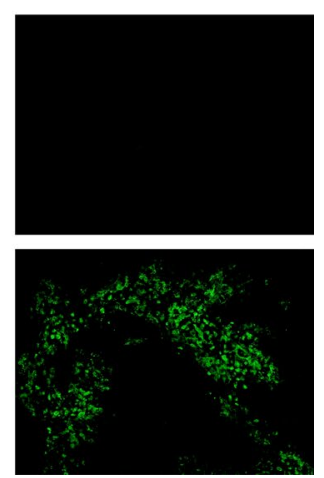

Infected 


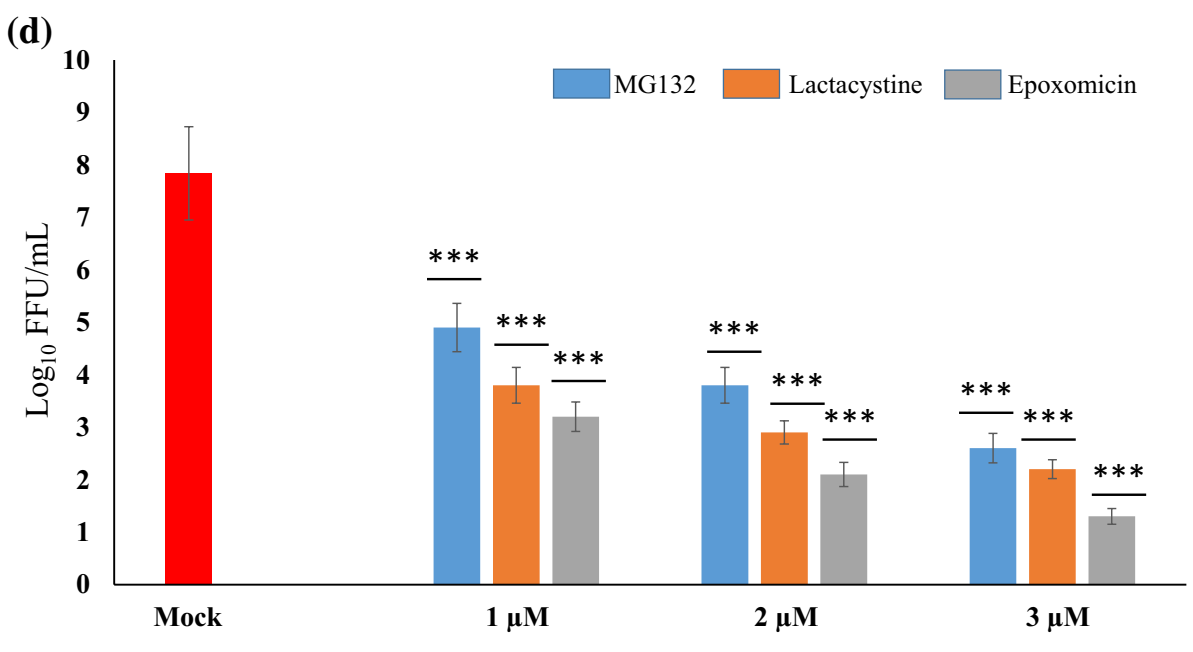

Chemical concentration

(e)

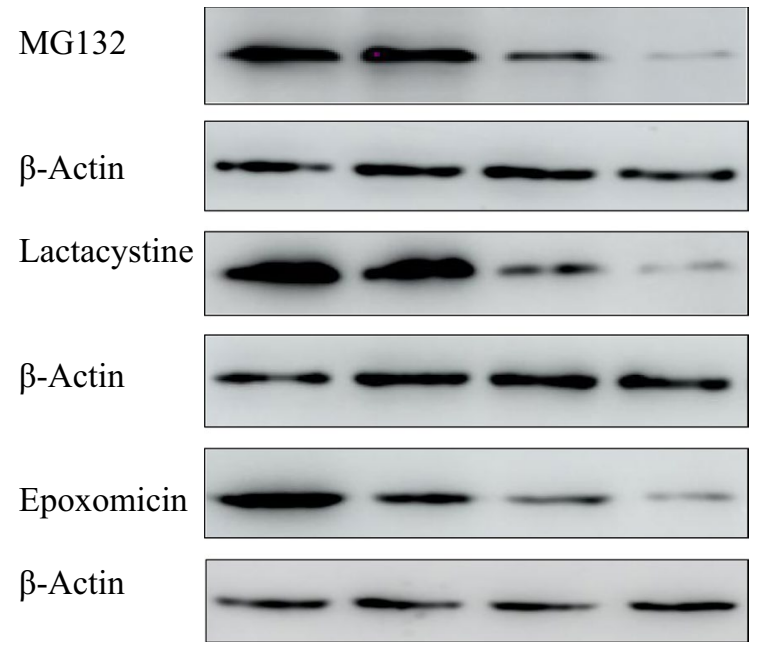

(f)

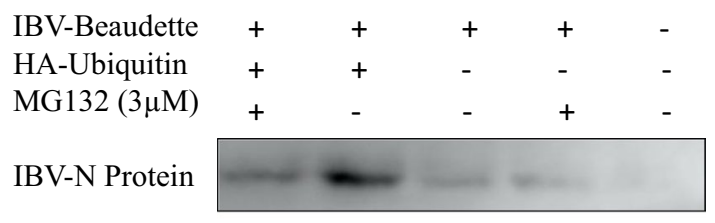

IB : anti-HA

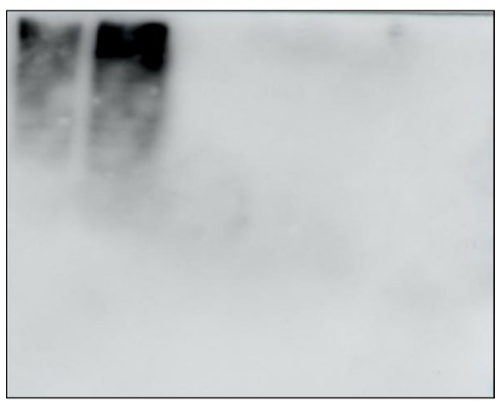

$\beta$-Actin

Fig. 1 (continued)

Fig. 2 Activation of the IFN response in DF-1 cells infected with cell-culture-adapted IBV strain M41. (a and b) Adaptation of the IBV M41 strain in DF-1 cells by repeated passaging. Replication was measured after the passage by IFA, using an antibody against the IBV membrane protein. (c) Increased expression of IFN $\beta$ transcripts in IBV (MOI of 10)-infected DF-1 cells detected by RT-qPCR. (d) Stimulation of IFN $\beta$ promoter activity after infection of IBV. DF-1 cells were infected with IBV, and after $24 \mathrm{~h}$ of infection, they were cotransfected with the ch-IFN $\beta$-luc reporter plasmid and the Renilla luciferase reporter plasmid. IFN $\beta$ promoter activity was measured by luciferase assay. The graphs represent the averages of three independent experiments. The error bars indicate standard error. (e) Replication of IBV is inhibited in the presence of an IFN response. Cells were infected with IBV, and after $18 \mathrm{~h}$ of infection, cells were either left untreated or transfected with poly(I:C). Cells were harvested after 24, 48, and $72 \mathrm{~h}$ of infection and lysed for protein extraction. An immunoblot was performed for detection of the IBV nucleocapsid and the cellular actin protein. (f) Expression of IRF3 is marginally upregulated in the presence of IBV replication. Cells were infected with IBV and, after $18 \mathrm{~h}$ of infection, transfected with poly(I:C) or left untreated. After $72 \mathrm{~h}$ of infection, cells were lysed for protein extraction. An immunoblot was performed for detection of the IBV nucleocapsid protein and chicken IRF3

cells with the plasmid pCMV-HA-Ubiquitin to overexpress ubiquitin. Western blotting was performed to detect hemagglutinin (HA)-tagged ubiquitin in the transfected cells using an anti-HA monoclonal antibody, and virus replication was detected using an antibody against the IBV nucleocapsid protein. The results showed that there was a higher level of IBV replication in the cells transfected with pCMV-HAUbiquitin than in untransfected control cells (Fig. 1f). 
(a)

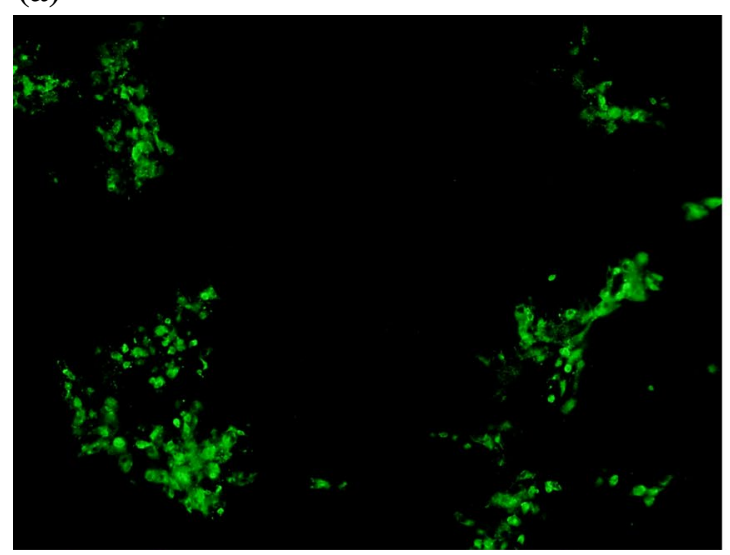

$10 \mathrm{X}$ Magnification for plaque counting

(c)

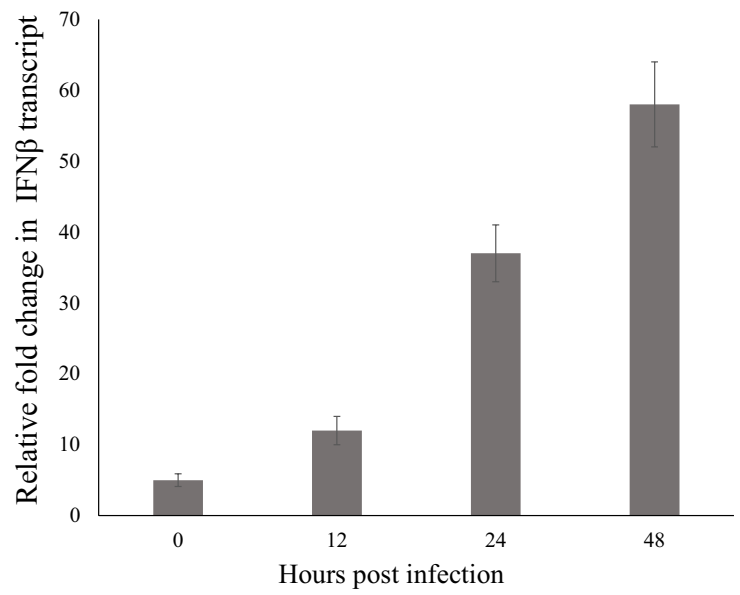

Reverse transcription-quantitative PCR

(e)

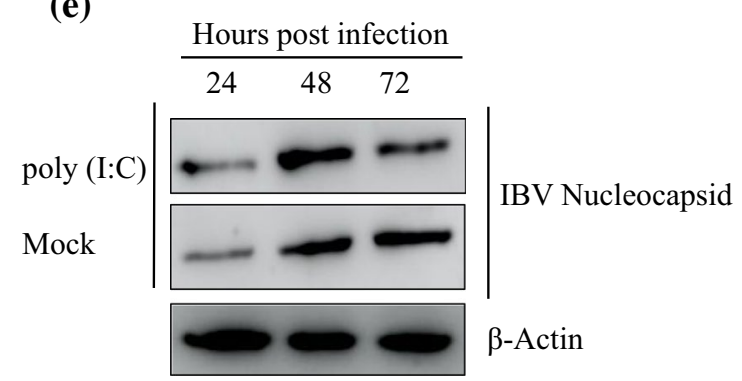

\section{IBV replication is inhibited by increased IFN $\beta$ expression induced by poly $(\mathrm{I}: \mathrm{C})$ transfection}

To test whether IBV replication could be inhibited by interferon induction, we monitored IBV replication in the presence of interferon induced by poly(I:C). Since Vero cells are (b)

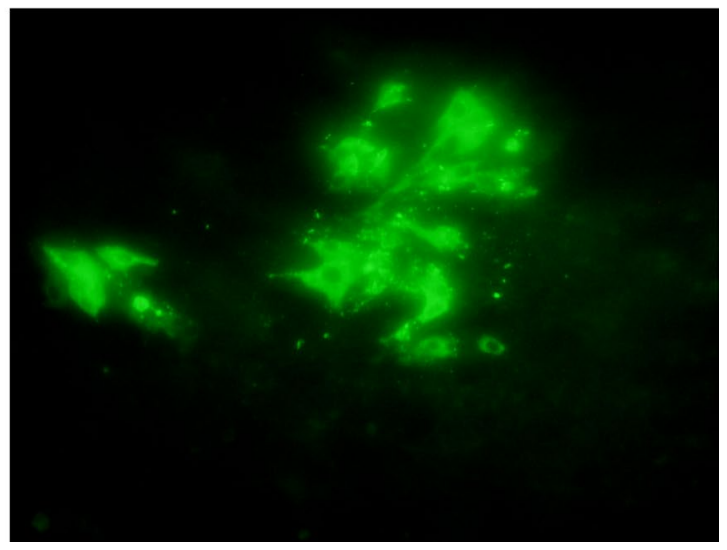

20 X Magnification

(d)

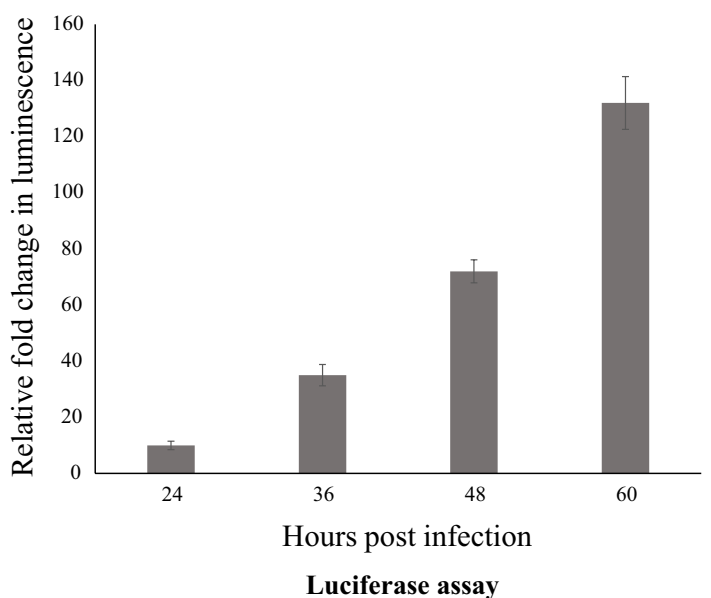

(f)

$\begin{array}{lllll}\mathrm{IBV} & - & - & + & + \\ \operatorname{poly}(\mathrm{I}: \mathrm{C}) & - & + & - & +\end{array}$

IBV Nucleocapsid

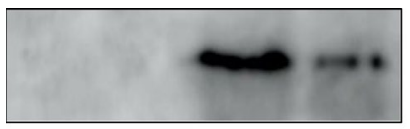

IRF3

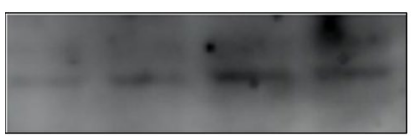

$\beta$-Actin

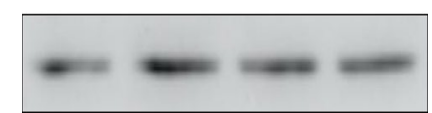

not suitable for studying immune response pathways and are of monkey rather than poultry origin, we used a pathogenic strain of IBV M41 and adapted it to chicken fibroblast (DF1) cells by repeated passaging. After 10 consecutive passages, infected DF-1 cells were immunostained to confirm the replication of IBV M41 (Fig. 2a and b), and the titer of 
IBV was measured as $\log _{10} \mathrm{FFU} / \mathrm{mL}=4.34$. DF-1 cells were infected with the culture-adapted IBV M41 strain at an MOI of 10 and harvested at different time points to measure the expression of IFN $\beta$ mRNA by RT-qPCR and IFN $\beta$ promoter activity by luciferase assay (Fig. $2 \mathrm{c}$ and d). Our results showed that there was a time-dependent increase in synthesis of IFN $\beta$ as virus replication progressed. This is in agreement with several previous reports showing that the IBV M41 strain elicits a type 1 innate immune response [9, 10]. Next, we studied the effect of IFN on the replication of IBV. There is a delayed IFN $\beta$ response after IBV infection [25]. We transfected DF-1 cells with poly(I:C) after $24 \mathrm{~h}$ of infection with IBV to stimulate IFN synthesis. Detection of IBV nucleocapsid protein by Western blot showed that there was reduction in IBV replication in the cells actively synthesizing IFNs induced by the synthetic analog poly(I:C) (Fig. 2e). We observed only a minor change in the expression of the IRF3 protein, presumably because we detected its expression using a polyclonal rabbit serum and not an $\mathrm{mAb}$ against the chicken p-IRF3, which is unavailable commercially. Nevertheless, there was a visible difference in its expression level in the presence and absence of poly(I:C) and IBV, indicating that IBV causes a decrease in IRF3 synthesis in the presence of interferon (Fig. 2f). Taken together, our results demonstrate that the cell-culture-adapted IBV M41 strain elicits a type 1 innate immune response, and in the presence of IFN, its replication is inhibited.

\section{The IBV papain-like protease domain (PLpro) inhibits IFN production}

The papain-like proteases of several coronaviruses have the property of suppressing the type 1 IFN response. This property is mediated by diverse mechanisms [26, 27]. To study the role of IBV PLpro in regulating the host innate immune response, we made the clone pCMV-N-myc-IBV PLpro and used it to transfect DF-1 cells. Its expression was confirmed by Western blot using an anti-myc antibody (data not shown). To rule out the possibility of a cytotoxic effect of PLpro, we performed a cell viability assay with DF-1 cells transfected with empty vector as well as the PLpro expression construct. We found that there was $<10 \%$ cytotoxicity in the PLproexpressing DF-1 cells, indicating that PLpro expression does not have a deleterious effect on the viability of the cells (Fig. 3a). Next, we examined the effect of IBV PLpro on IFN expression. After $48 \mathrm{~h}$ of transfection, DF-1 cells were transfected with poly(I:C) to stimulate IFN induction. RT-qPCR was performed after $6 \mathrm{~h}$ of poly(I:C) stimulation to determine the IFN $\beta$ mRNA level. IBV PLpro expression caused a significant reduction ( $\sim 3.5$-fold) in IFN $\beta$ mRNA expression when compared with the cells transfected with empty vector (Fig. 3b). This observation also confirmed that the reduced expression of IFN $\beta$ mRNA in the PLpro-expressing cells was due to the antagonist effect of the IBV PLpro enzyme. We also measured IFN $\beta$ promoter activity in the presence of pCMV-N-myc-IBV PLpro by transfection with the IFN $\beta$ luciferase reporter plasmid. The results of the reporter assay showed that IBV PLpro significantly inhibited ( 3.8 -fold) the poly(I:C)-induced IFN $\beta$ promoter activation (Fig. 3c). Since overexpression of signal molecules such as MDA5 and TBK1 leads to the activation of the IFN $\beta$ promoter $[28,29]$, DF-1 cells were transfected with plasmids encoding the IFN $\beta$ reporter, PLpro, and the specific components of the signaling pathway, MDA5 and TBK1, to confirm the IFN antagonist property of the IBV PLpro enzyme. The results showed that IBV PLpro indeed inhibited the IFN $\beta$ promoter activity in the presence of the MDA5 and TBK1 proteins by $\sim 1.8$ - and $\sim 2.1$-fold, respectively (Fig. 3d and e). Taken together, our data indicate that IBV PLpro functions as an antagonist of type I IFN induction.

\section{IBV PLpro deubiquitinates both MDA5 and TBK1}

Based on the results described above, indicating that active functioning of the UPS is essential for IBV replication and that its PLpro domain has IFN antagonist activity, we aimed to further understand the specific role of IBV PLpro in deubiquitination of cellular proteins of innate immune response pathways that are post-translationally modified by the UPS. Due to the absence of RIG-I in chicken, MDA5 acts as a primary sensor for IBV dsRNA, and there is an elevated expression of IFN $\beta[10,30]$. We reasoned that IBV PLpro could potentially lead to deubiquitination of both MDA5 and TBK1, as ubiquitination of these two molecules is required for their activation $[13,14]$. Due to the very low level of expression of endogenous MDA5 and TBK1 and the unavailability of chicken antibodies against both of these proteins, we decided to coexpress them along with IBV PLpro in DF-1 cells and perform the ubiquitination assay after coimmunoprecipitation. We cloned chicken MDA5 and TBK1 into the pCMV-N-Flag vector. DF-1 cells were cotransfected with pCMV-N-myc-IBV PLpro and pCMV-N-Flag-MDA5 or pCMV-N-Flag-TBK1. After $48 \mathrm{~h}$ of transfection, cells were harvested, and co-immunoprecipitation was carried out using an anti-Flag antibody. A Western blot was performed using an anti-ubiquitin antibody, which can detect all cellular proteins, including MDA5 and TBK1, that are posttranslationally modified by ubiquitin tagging. The results showed that IBV PLpro efficiently deubiquitinates MDA5 and TBK1 along with other cellular proteins, which was not observed in cells transfected with the empty pCMV-N-myc vector (Fig. 4a and c), while the overall MDA5 and TBK1 expression levels in the whole-cell lysate were not affected (Fig. 4b and d). These results suggest that IBV PLpro may inhibit MDA5 and TBK1 activation via its deubiquitinase activity. 


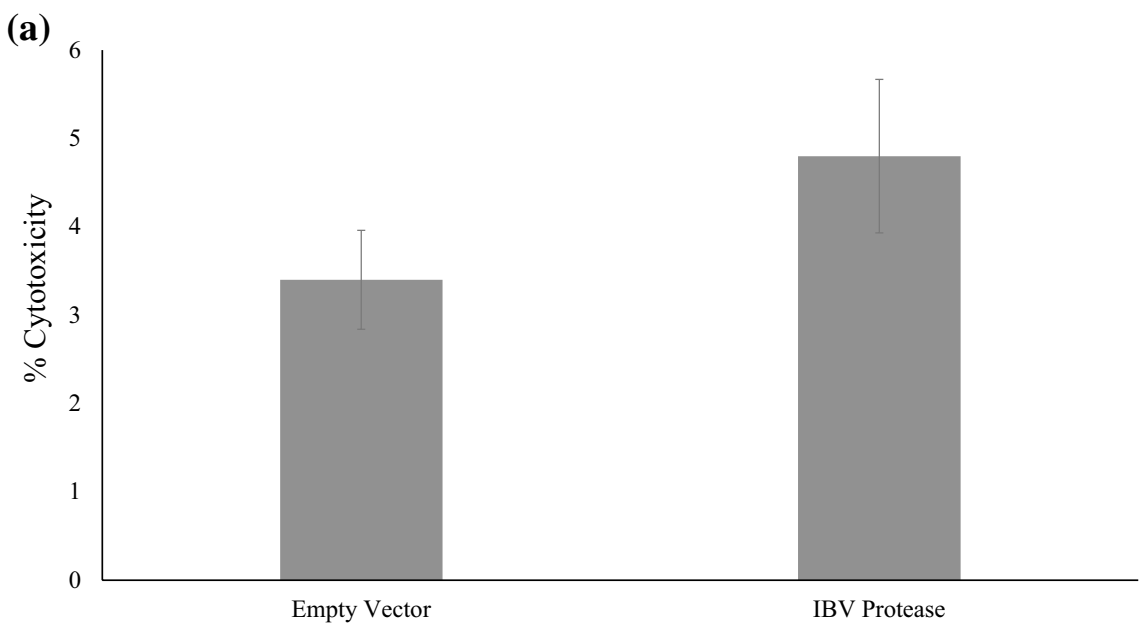

(b)

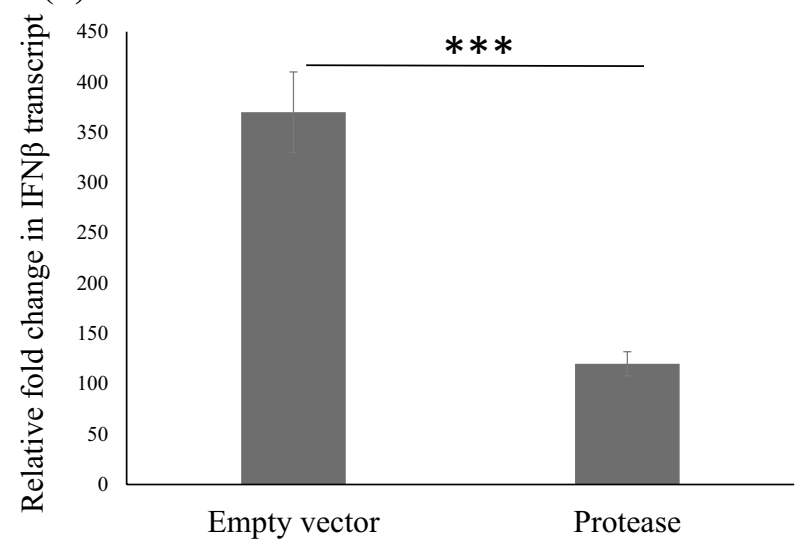

Reverse transcription-quantitative PCR (c)

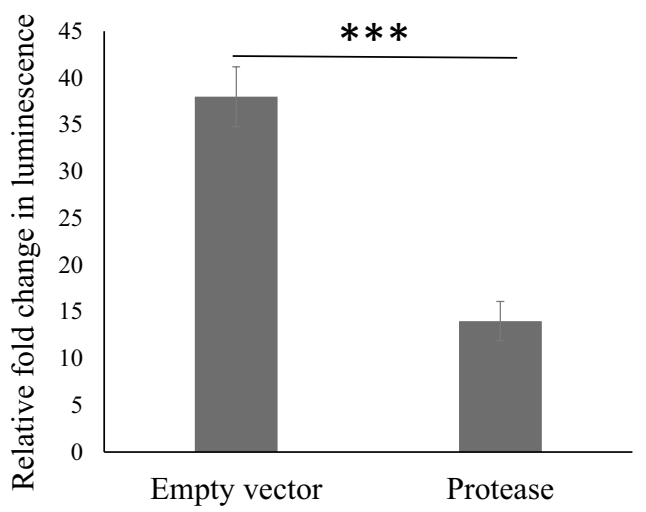

Luciferase assay

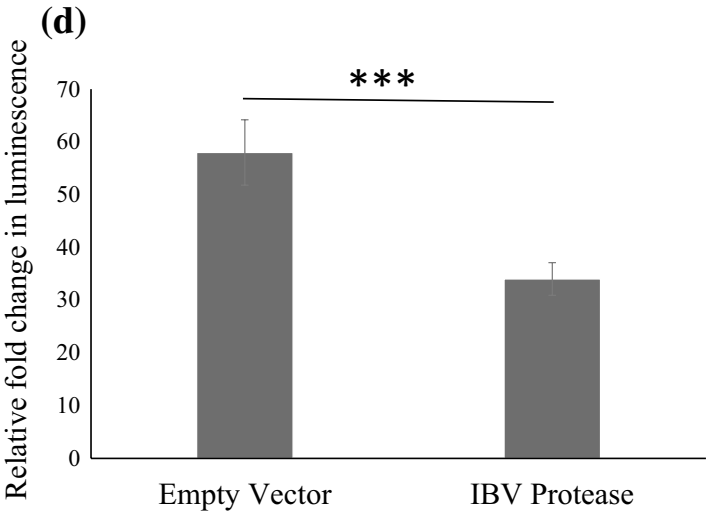

MDA-5

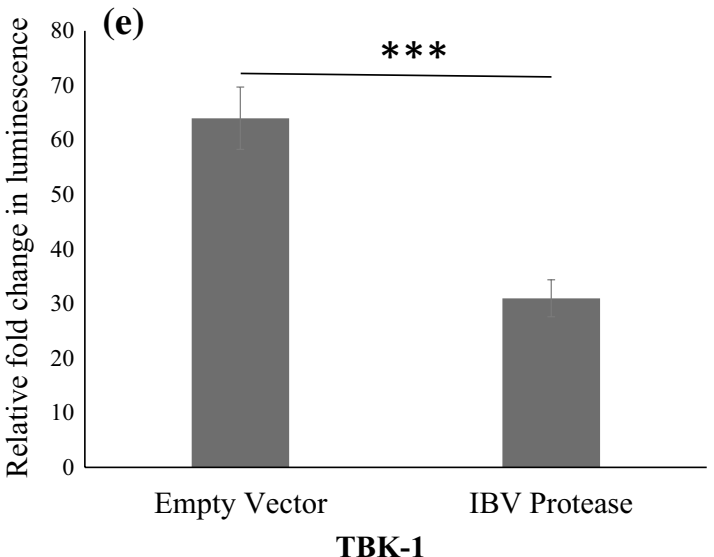

TBK-1

Fig. 3 IBV PLpro is an IFN antagonist. (a) The cell viability after PLpro transfection was assessed using an alamarBlue assay. (b) PLpro reduces the expression of IFN $\beta$ mRNA expression induced by poly(I:C). DF-1 cells were transfected with either empty vector (EV) or pCMV-N-mycIBV PLpro. After $36 \mathrm{~h}$ of transfection, cells were again transfected with poly(I:C) to induce IFN $\beta$ mRNA expression. After $12 \mathrm{~h}$ of poly(I:C) treatment, RT-qPCR was performed to detect IFN $\beta$ transcripts. (b-d) IBV PLpro inhibits IFN $\beta$ promoter activity. DF-1 cells were transfected with either empty vector (EV) or pCMV-N-myc-IBV PLpro plasmid, along with the IFN $\beta$ luciferase reporter and Renilla luciferase plasmid. This step is termed as "first transfection". (b) The cells were transfected with poly(I:C) $48 \mathrm{~h}$ after the first transfection. Luciferase activity was assayed $12 \mathrm{~h}$ after poly(I:C) transfection. (c) Cells were transfected with pCMV-N-Flag-MDA5 and (d) pCMV-N-Flag-TBK1 $24 \mathrm{~h}$ after the first transfection. Luciferase activity was assayed $24 \mathrm{~h}$ after the second transfection. Luminescence values are shown as a relative level (fold) in comparison with the EV control. The graphs represent the averages of three independent experiments. The error bars indicate standard error. Significant differences between test samples and controls are indicated by asterisks $(* * *, p \leq 0.005)$ 


\section{IBV replication in DF-1 cells inhibits ubiquitination of MDA5 and TBK1}

The results described above showed that the overexpression of IBV PLpro in DF-1 cells causes deubiquitination of MDA5 and TBK1. To our knowledge, no other IBV protein has deubiquitinase activity except the PLpro enzyme. This leads to the conclusion that IBV PLpro expressed after proteolytic processing of the orf1-encoded polyprotein during virus infection could also perform the deubiquitinase function. To test this assumption, we infected DF- 1 cells with the cell-culture-adapted IBV M41 strain at an MOI of 10, and at $24 \mathrm{hpi}$, we transfected these cells with plasmids for overexpression of MDA5 and TBK1. At $48 \mathrm{~h}$ post-transfection, the cells were harvested, and whole-cell lysates were subjected to immunoprecipitation using anti-Flag antibody. After immunoprecipitation, protein samples were probed with an anti-ubiquitin antibody to measure the ubiquitination level of cellular proteins. The results showed that both MDA5 and TBK1 had lower ubiquitination levels in DF-1 cells infected with IBV than in the uninfected control cells (Fig. 5a and c). There was no difference between the control and infected samples in the total ubiquitination level in whole-cell lysates (Fig. 5b and d), demonstrating that IBV replication inhibited the ubiquitination of MDA5 and TBK1, presumably from its own PLpro enzyme synthesized by open reading frame 1.

\section{Discussion}

IBV is a highly contagious virus pathogen of poultry that causes severe damage to the trachea and kidney. Its pathogenicity varies according to the genotype and strain [31]. An active ubiquitin proteasome system (UPS) is essential for the replication of many viruses, and inhibitors of the UPS are also known to inhibit virus replication [32]. Viruses modulate the host cell UPS for their survival [33]. In the present study, we investigated the role of the UPS in the replication of IBV and its cross-talk with the innate immune system during IBV infection. We also examined the role of the IBV papain-like protease (PLpro) domain in regulating both of these pathways.

In order to ascertain the role of the UPS in IBV replication, we used three different UPS inhibitors, namely, MG132, lactacystin, and epoxomicin, to determine their effect on the replication of the IBV Beaudette strain in Vero cells. All three inhibitors tested in this study caused a significant reduction in the level of virus replication, and the degree of inhibition was proportional to the dose applied. MG132 reduces the pool of free ubiquitin in cells. In the case of human parainfluenza virus 5, MG132 inhibits virus budding by depleting the cellular pool of free ubiquitin [34]. Therefore, to determine whether the observed inhibition of

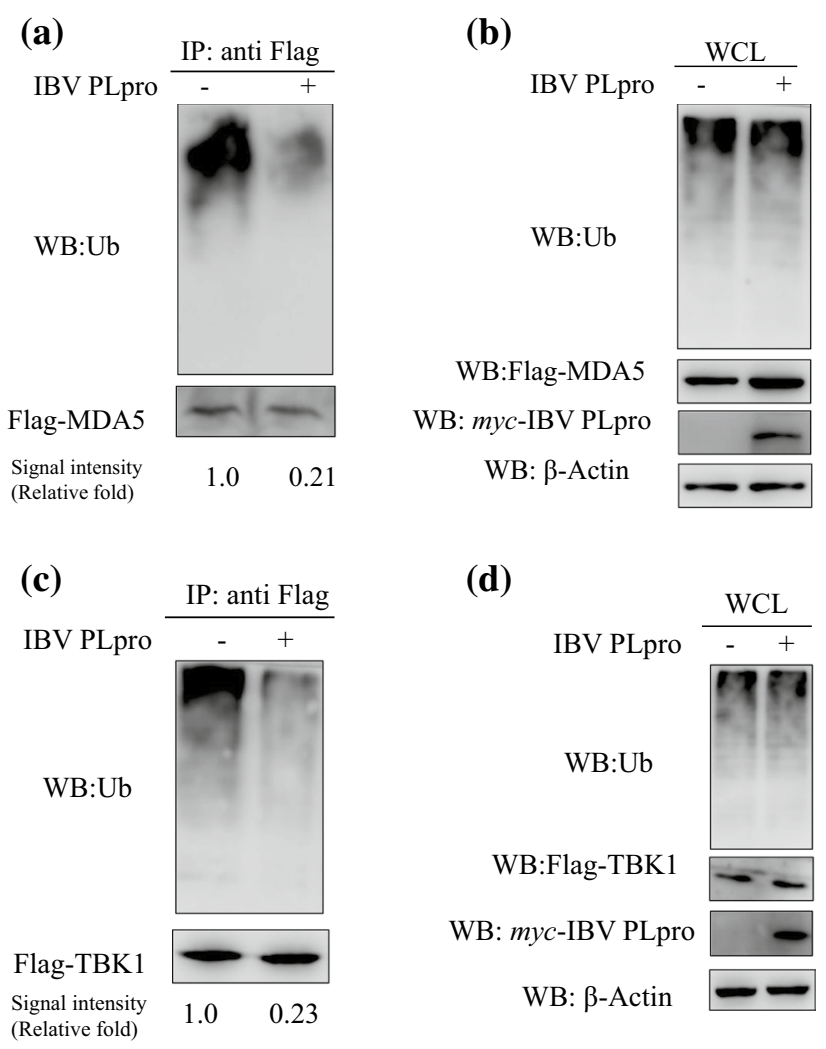

Fig. 4 Type-I-IFN-response-pathway-related proteins MDA5 and TBK1 are deubiquitinated by IBV PLpro. DF-1 cells were transfected with the empty vector (EV) or pCMV-N-myc-IBV PLpro. After $24 \mathrm{~h}$ of transfection, the cells were transfected with pCMV-N-Flag-MDA5 (a) or the plasmid pCMV-N-Flag-TBK1 (c). After $72 \mathrm{~h}$ of PLpro plasmid transfection, cells were lysed for protein extraction. Immunoprecipitation was done using an anti-Flag antibody. Eluted proteins were probed to measure the ubiquitination of cellular proteins using an anti-ubiquitin antibody. The relative ubiquitination signal intensity is shown below the blots after normalization with MDA5 (a) and TBK1 (c). (b and d) Prior to immunoprecipitation, Western blotting of whole-cell lysate was done using the corresponding antibodies for the confirmation of protein expression from the indicated plasmids

IBV replication by MG132 was due to depletion of free ubiquitin, we expressed ubiquitin ectopically by transient transfection with the plasmid pCMV-HA-Ubiquitin, which partially restored IBV replication even in the presence of the inhibitor MG132. This confirms that the active functioning of the UPS is important for IBV replication.

For many coronaviruses, it has been reported that type 1 IFNs, such as IFN $\alpha$ and IFN $\beta$, are important for the host cell's innate immune response against infection and also modulate adaptive immunity. Viruses employ different strategies to evade the host immune response. The interferon (IFN) response mediated by IFN-stimulated genes (ISGs) makes cells resistant to pathogens. After IBV infection, there is an increase in the synthesis of diverse cytokines and chemokines, which increases the resistance and survival of the cells $[35,36]$. Previously, it was shown that, upon IBV 

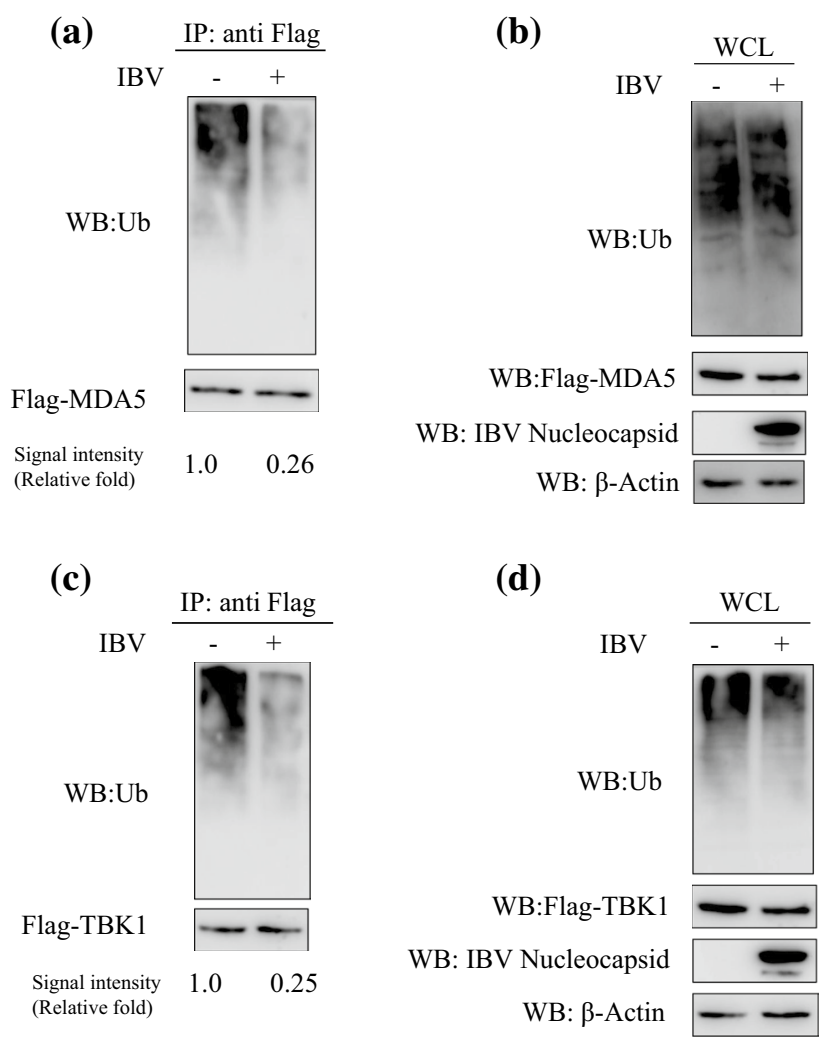

Fig. 5 Type-I-IFN-response-pathway-related proteins MDA5 and TBK1 are deubiquitinated by IBV. DF-1 cells were either infected with IBV M41 at an MOI of 10 or left uninfected. After $24 \mathrm{~h}$ of infection, cells were transfected with the plasmid pCMV-N-Flag-MDA5 (a) or pCMV-N-Flag-TBK1 (c). After $72 \mathrm{~h}$ of infection, cells were lysed for protein extraction. Immunoprecipitation was done using an anti-Flag antibody. Eluted proteins were probed for measurement of ubiquitination of cellular proteins using an anti-ubiquitin antibody. The relative ubiquitination signal intensity is shown below the blots after normalization with MDA5 (a) and TBK1 (c). (b and d) Prior to immunoprecipitation, Western blotting of whole cell lysate was done using the corresponding antibodies for confirmation of protein expression from the indicated plasmids as well as viral replication

infection, there is an increase in the synthesis of type 1 IFN. TLR3 and MDA5 recognize the double-stranded RNA intermediate synthesized during the early stage of IBV infection [10]. MDA5, together with the MAVS protein makes a cascade with other adapter molecules such as IKKe and TANK binding kinase 1 (TBK1), which activate IRF3 by phosphorylation, leading to the synthesis of IFN $\beta[37,38]$. Ubiquitination of MDA5 and TBK1 is essential for their activation, and their deubiquitination by virus-encoded proteins renders them unavailable for IFN signaling [13-15]. The results of our study demonstrate that IBV induces the synthesis of IFN $\beta$ in a time-dependent manner and that PLpro of IBV is an IFN antagonist whose activity is enhanced when MDA5 and TBK1 are overexpressed (Fig. 6).

Papain-like proteases of viruses belonging to the order Nidovirales are known to be involved in proteolytic

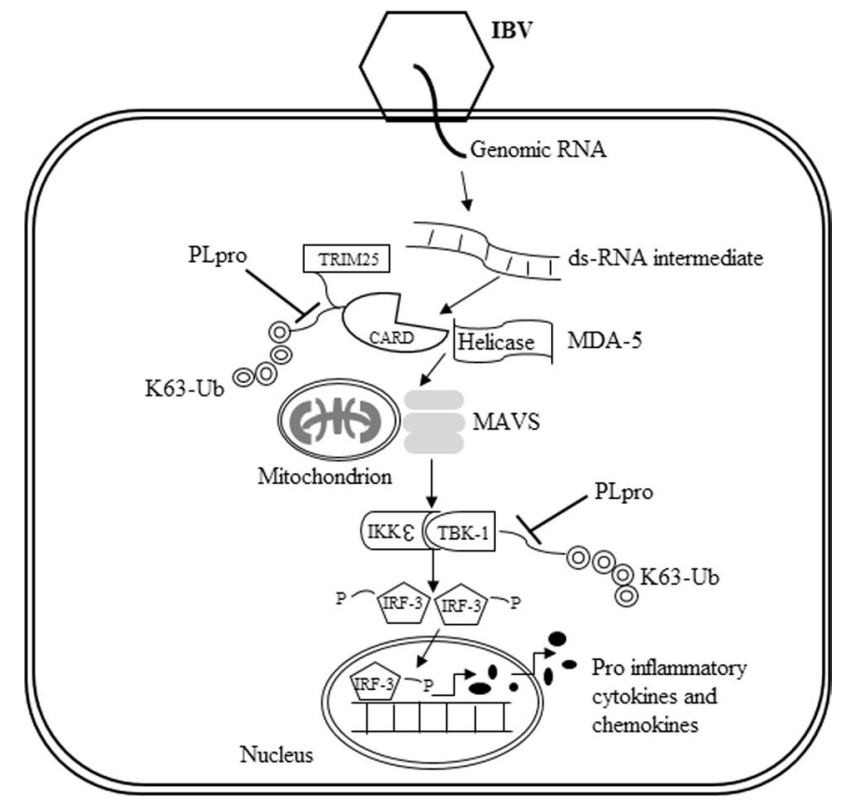

Fig. 6 Schematic representation of how IBV PLpro inhibits the IFN synthesis

processing, deubiquitination (DUB), and deISGylation [39]. Similar to those of other coronaviruses, the PLpro of IBV is also primarily responsible for proteolytic processing of the nonstructural polyprotein encoded by open reading frame 1 [6]. It also possesses in vitro deubiquitinase activity, which involves the processing of both K48- and K63-linked polyubiquitin chains $[7,8]$. The PLpro proteins of severe acute respiratory syndrome coronavirus (SARS-CoV) and IBV have considerable sequence similarity in their catalytic core region, which is responsible for proteolytic activity, as well as the conserved "DUB signature residues", which recognize substrates with the sequence LXGG [7, 40]. PLpro enzymes of members of the order Nidovirales also have IFN antagonist properties, which are mediated by their deubiquitinase activity [39]. Ubiquitination and deubiquitination are important for the functioning of the innate immune response pathway [41]. The deISGylating and DUB activity of PLpro of many coronaviruses has been known for a long time, but the exact mechanism of PLpro-mediated antagonism of the innate immune response is still not fully understood [42]. PLpro from Middle East respiratory syndrome coronavirus (MERS-CoV) has been shown to degrade the polyubiquitin chain associated with MDA5 and RIG-I, preventing activation of host antiviral signaling pathways [43]. The IFN antagonist activity of MHV-A59-encoded PLP2 involves its binding to IRF3, its deubiquitination, and finally, prevention of its nuclear translocation. This protein also deubiquitinates TBK1 and reduces its kinase activity, resulting in the inhibition of IFN $\beta$ reporter activity $[44,45]$. 
Accessory proteins of IBV play a role in inhibiting the host IFN response [46, 47]. In this report, we show that PLpro of IBV is also an IFN antagonist. It inhibits the expression of IFN $\beta$ mRNA as well as protein. It also deubiquitinates MDA5 and TBK1 because ubiquitination of both of these proteins is essential for their activation [15]. A reduced level of ubiquitination of MDA5 and TBK1 was also observed in the case of IBV M41 replication in DF-1 cells, presumably by the functional PLpro encoded by IBV open reading frame 1 . SARS-CoV PLpro has the property of cleaving ubiquitin- and ISG15-conjugated proteins and thereby helps the virus to subvert host innate immunity. Chemical inhibitors targeting the virus-specific PLpro, without affecting its deubiquitinase activity, inhibit virus replication, suggesting that specific inhibitors of the viral deubiquitinase activity will also be useful against virus infection. Previously, chemical inhibitors targeting the $\mathrm{CoV}$ papain-like proteases have been useful for inhibiting virus replication [48]. Bortezomib is an FDA-approved proteasome inhibitor that can be useful in the treatment of multiple myeloma [49]. So far, there is no chemoprophylactic drug available commercially for IBV infection. Our results suggest that IBV PLpro can be an attractive target for designing antivirals against IBV infection.

\section{Conclusion}

Our study shows for the first time that an active ubiquitin proteasome system is essential for efficient replication of IBV and that viral nsp3-encoded PLpro enzyme deubiquitinates MDA5 and TBK1. Both of these proteins are constituents of the signaling cascade responsible for IBV-induced increased synthesis of type 1 interferons. Deubiquitination of MDA5 and TBK1 might be responsible for the IFN antagonist activity of the IBV PLpro enzyme. Hence, IBV PLpro might serve as a potential target for development of chemical therapeutics against IBV infection. This study will also be helpful for understanding the role of nonstructural proteins in IBV replication and pathogenesis.

Acknowledgements This work was supported by the National Key Research and Development Program of China (Grant no. 2016YFD0500800) and the China Agriculture Research System (Grant no. CARS-40-K13).

Author contributions NKO, ML and JYZ designed the research plan. $\mathrm{NKO}$ performed the experiments, analyzed the data and drafted the manuscript. JJL, TQY and CXF assisted in doing the experiments.

\section{Declarations}

Conflict of interest The authors declare that there is no conflict of interest.

\section{References}

1. Cavanagh D (2007) Coronavirus avian infectious bronchitis virus. Vet Res 38(2):281-297. https://doi.org/10.1051/vetres:2006055

2. Legnardi M, Tucciarone CM, Franzo G, Cecchinato M (2020) Diagnosis and control. Vet Sci 7(2):E79. https://doi.org/10.3390/ vetsci7020079

3. Gorbalenya AE, Enjuanes L, Ziebuhr J, Snijder EJ (2006) Nidovirales: evolving the largest RNA virus genome. Virus Res 117(1):17-37. https://doi.org/10.1016/j.virusres.2006.01.017

4. Brian DA, Baric RS (2005) Coronavirus genome structure and replication. Curr Top Microbiol Immunol 287:1-30. https://doi. org/10.1007/3-540-26765-4_1

5. Lei J, Kusov Y, Hilgenfeld R (2018) Nsp3 of coronaviruses: structures and functions of a large multi-domain protein. Antiviral Res 149:58-74. https://doi.org/10.1016/j.antiviral.2017.11.001

6. Ng LF, Liu DX (1998) Identification of a 24-kDa polypeptide processed from the coronavirus infectious bronchitis virus 1 a polyprotein by the 3C-like proteinase and determination of its cleavage sites. Virology 243(2):388-395. https://doi.org/10.1006/ viro. 1998.9058

7. Kong L, Shaw N, Yan L, Lou Z, Rao Z (2015) Structural view and substrate specificity of papain-like protease from avian infectious bronchitis virus. J Biol Chem 290(11):7160-7168. https://doi.org/ 10.1074/jbc.M114.628636

8. Yu L, Zhang X, Wu T et al (2017) The papain-like protease of avian infectious bronchitis virus has deubiquitinating activity. Arch Virol 162(7):1943-1950. https://doi.org/10.1007/ s00705-017-3328-y

9. Barjesteh N, O'Dowd K, Vahedi SM (2020) Antiviral responses against chicken respiratory infections: Focus on avian influenza virus and infectious bronchitis virus. Cytokine 127:154961. https://doi.org/10.1016/j.cyto.2019.154961

10. Kint J, Fernandez-Gutierrez M, Maier HJ et al (2015) Activation of the chicken type I interferon response by infectious bronchitis coronavirus. J Virol 89(2):1156-1167. https://doi.org/10.1128/ JVI.02671-14

11. Gao G, Luo H (2006) The ubiquitin-proteasome pathway in viral infections. Can J Physiol Pharmacol 84(1):5-14. https://doi.org/ 10.1139/y05-144

12. Zheng Y, Gao C (2020) Fine-tuning of antiviral innate immunity by ubiquitination. Adv Immunol 145:95-128. https://doi.org/10. 1016/bs.ai.2019.11.004

13. Lang X, Tang T, Jin T et al (2017) TRIM65-catalized ubiquitination is essential for MDA5-mediated antiviral innate immunity. $\mathrm{J}$ Exp Med 214:459-473. https://doi.org/10.1084/jem.20160592

14. Ikeda F, Hecker CM, Rozenknop A et al (2007) Involvement of the ubiquitin-like domain of TBK1/IKK-i kinases in regulation of IFN-inducible genes. EMBO J 26(14):3451-3462. https://doi.org/ 10.1038/sj.emboj.7601773

15. Brisse M, Ly H (2019) Comparative structure and function analysis of the RIG-I-like receptors: RIG-I and MDA5. Front Immunol 10:1586. https://doi.org/10.3389/fimmu.2019.01586

16. Binns MM, Boursnell ME, Tomley FM, Brown DK (1986) Comparison of the spike precursor sequences of coronavirus IBV strains M41 and 6/82 with that of IBV Beaudette. J Gen Virol 67(Pt 12):2825-2831. https://doi.org/10.1099/ 0022-1317-67-12-2825

17. Wu H, Yang H, Ji G et al (2019) SUMO1 modification facilitates avibirnavirus replication by stabilizing polymerase VP1. J Virol 93:e02227-e2318. https://doi.org/10.1128/JVI.02227-18

18. Ye C, Jia L, Sun Y et al (2014) Inhibition of antiviral innate immunity by birnavirus VP3 protein via blockage of viral double-stranded RNA binding to the host cytoplasmic RNA detector 
MDA5. J Virol 88(19):11154-11165. https://doi.org/10.1128/JVI. 01115-14

19. Livak KJ, Schmittgen TD (2001) Analysis of relative gene expression data using real-time quantitative PCR and the 2(-Delta Delta C(T)) Method. Methods 25(4):402-408. https://doi.org/10.1006/ meth.2001.1262

20. Payne AF, Binduga-Gajewska I, Kauffman EB, Kramer LD (2006) Quantitation of flaviviruses by fluorescent focus assay. J Virol Methods 134(1-2):183-189. https://doi.org/10.1016/j.jviromet. 2006.01.003

21. Raaben M, Posthuma CC, Verheije MH et al (2010) The ubiquitinproteasome system plays an important role during various stages of the coronavirus infection cycle. J Virol 84(15):7869-7879. https://doi.org/10.1128/JVI.00485-10

22. Yu GY, Lai MM (2005) The ubiquitin-proteasome system facilitates the transfer of murine coronavirus from endosome to cytoplasm during virus entry. J Virol 79(1):644-648. https://doi.org/ 10.1128/JVI.79.1.644-648.2005

23. Kumar P, Nagarajan A, Uchil PD (2018) Analysis of cell viability by the alamarblue assay. Cold Spring Harb Protoc 2018:6. https:// doi.org/10.1101/pdb.prot095489

24. López T, Silva-Ayala D, López S, Arias CF (2011) Replication of the rotavirus genome requires an active ubiquitin-proteasome system. J Virol. 85(22):11964-11971. https://doi.org/10.1128/JVI. 05286-11

25. Jang HJ, Song KD (2020) Expression patterns of innate immunity-related genes in response to polyinosinic:polycytidylic acid (poly[I:C]) stimulation in DF-1 chicken fibroblast cells. J Anim Sci Technol 62(3):385-395. https://doi.org/10.5187/jast.2020. 62.3.385

26. Chen X, Yang X, Zheng Y et al (2014) SARS coronavirus papain-like protease inhibits the type I interferon signaling pathway through interaction with the STING-TRAF3-TBK1 complex. Protein Cell 5(5):369-381. https://doi.org/10.1007/ s13238-014-0026-3

27. Devaraj SG, Wang N, Chen Z et al (2007) Regulation of IRF3-dependent innate immunity by the papain-like protease domain of the severe acute respiratory syndrome coronavirus. J Biol Chem 282(44):32208-32221. https://doi.org/10.1074/jbc.M704870200

28. Kawai T, Takahashi K, Sato $S$ et al (2005) IPS-1, an adaptor triggering RIG-I- and Mda5-mediated type I interferon induction. Nat Immunol 6(10):981-988. https://doi.org/10.1038/ni1243

29. Lu LL, Puri M, Horvath CM, Sen GC (2008) Select paramyxoviral $\mathrm{V}$ proteins inhibit IRF3 activation by acting as alternative substrates for inhibitor of kappaB kinase epsilon (IKKe)/TBK1. J Biol Chem 283(21):14269-14276. https://doi.org/10.1074/jbc. M710089200

30. Karpala AJ, Stewart C, McKay J, Lowenthal JW, Bean AG (2011) Characterization of chicken Mda5 activity: regulation of IFN- $\beta$ in the absence of RIG-I functionality. J Immunol 186(9):5397-5405. https://doi.org/10.4049/jimmunol.1003712

31. Lin SY, Chen HW (2017) Infectious bronchitis virus variants: molecular analysis and pathogenicity investigation. Int J Mol Sci 18(10):2030. https://doi.org/10.3390/ijms 18102030

32. Choi AG, Wong J, Marchant D, Luo H (2013) The ubiquitinproteasome system in positive-strand RNA virus infection. Rev Med Virol 23(2):85-96. https://doi.org/10.1002/rmv.1725

33. Luo $H$ (2016) Interplay between the virus and the ubiquitin-proteasome system: molecular mechanism of viral pathogenesis. Curr Opin Virol 17:1-10. https://doi.org/10.1016/j.coviro.2015.09.005

34. Schmitt AP, Leser GP, Morita E, Sundquist WI, Lamb RA (2005) Evidence for a new viral late-domain core sequence, FPIV, necessary for budding of a paramyxovirus. J Virol 79(5):2988-2997. https://doi.org/10.1128/JVI.79.5.2988-2997.2005
35. Chhabra R, Ball C, Chantrey J, Ganapathy K (2018) Differential innate immune responses induced by classical and variant infectious bronchitis viruses in specific pathogen free chicks. Dev Comp Immunol 87:16-23. https://doi.org/10.1016/j.dci.2018.04. 026

36. Pei J, Sekellick MJ, Marcus PI, Choi IS, Collisson EW (2001) Chicken interferon type I inhibits infectious bronchitis virus replication and associated respiratory illness. J Interferon Cytokine Res 21(12):1071-1077. https://doi.org/10.1089/107999001317205204

37. Fitzgerald KA, McWhirter SM, Faia KL et al (2003) IKKepsilon and TBK1 are essential components of the IRF3 signaling pathway. Nat Immunol 4(5):491-496. https://doi.org/10.1038/ni921

38. Wang Y, Yin Y, Lan X et al (2017) Molecular characterization, expression of chicken TBK1 gene and its effect on IRF3 signaling pathway. PLoS ONE 12(5):e0177608. https://doi.org/10.1371/ journal.pone.0177608

39. Mielech AM, Chen Y, Mesecar AD, Baker SC (2014) Nidovirus papain-like proteases: multifunctional enzymes with protease, deubiquitinating and deISGylating activities. Virus Res 194:184190. https://doi.org/10.1016/j.virusres.2014.01.025

40. Barretto N, Jukneliene D, Ratia K et al (2005) The papain-like protease of severe acute respiratory syndrome coronavirus has deubiquitinating activity. J Virol 79(24):15189-15198. https://doi. org/10.1128/JVI.79.24.15189-15198.2005

41. Chen M, Gerlier D (2006) Viral hijacking of cellular ubiquitination pathways as an anti-innate immunity strategy. Viral Immunol 19(3):349-362. https://doi.org/10.1089/vim.2006.19.349

42. Lei J, Hilgenfeld R (2017) RNA-virus proteases counteracting host innate immunity. FEBS Lett 591(20):3190-3210. https://doi. org/10.1002/1873-3468.12827

43. Yang X, Chen X, Bian G et al (2014) Proteolytic processing, deubiquitinase and interferon antagonist activities of Middle East respiratory syndrome coronavirus papain-like protease. J Gen Virol 95(Pt 3):614-626. https://doi.org/10.1099/vir.0.059014-0

44. Wang G, Chen G, Zheng D, Cheng G, Tang H (2011) PLP2 of mouse hepatitis virus A59 (MHV-A59) targets TBK1 to negatively regulate cellular type I interferon signaling pathway. PLoS ONE 6(2):e17192. https://doi.org/10.1371/journal.pone.0017192

45. Zheng D, Chen G, Guo B, Cheng G, Tang H (2008) PLP2, a potent deubiquitinase from murine hepatitis virus, strongly inhibits cellular type I interferon production. Cell Res 18(11):1105-1113. https://doi.org/10.1038/cr.2008.294

46. Kint J, Langereis MA, Maier HJ et al (2016) Infectious bronchitis coronavirus limits interferon production by inducing a host shutoff that requires accessory protein 5b. J Virol 90:7519-7528. https:// doi.org/10.1128/JVI.00627-16

47. Kint J, Dickhout A, Kutter J et al (2015) Infectious bronchitis coronavirus inhibits STAT1 signaling and requires accessory proteins for resistance to type I interferon activity. J Virol 89(23):1204712057. https://doi.org/10.1128/JVI.01057-15

48. Goyal B, Goyal D (2020) Targeting the dimerization of the main protease of coronaviruses: a potential broad-spectrum therapeutic strategy. ACS Comb Sci 22(6):297-305. https://doi.org/10.1021/ acscombsci.0c00058

49. Kouroukis CT, Fernandez LA, Crump M et al (2011) A phase II study of bortezomib and gemcitabine in relapsed mantle cell lymphoma from the National Cancer Institute of Canada Clinical Trials Group (IND 172) [published correction appears in Leuk Lymphoma. 2011 Jun;52(6):1160. Gascoyne, Randy D [added]]. Leuk Lymphoma 52(3):394-399. https://doi.org/10.3109/10428 194.2010.546015

Publisher's Note Springer Nature remains neutral with regard to jurisdictional claims in published maps and institutional affiliations. 